\title{
Back Propagation Neural Network for Defect Detection of Woven Fabrics
}

\author{
Gnanaprakash.V \\ Assistant Professor \\ Dhanalakshmi Srinivasan \\ College of Engineering \& \\ Technology, India
}

\author{
Sathishkumar.N \\ Assistant Professor \\ KPR Institute of Engineering \\ and Technology, India
}

\author{
Finney Daniel Shadrach S \\ Assistant Professor \\ KPR Institute of Engineering \\ and Technology, India
}

\begin{abstract}
Fabric defect detection plays a very important role for the automatic detection in fabrics. In this paper, fabric texture feature is extracted using Grey Level Co-occurrence Matrix (GLCM). The Co-occurrence matrices functions characterize the texture of an image by calculating how often pairs of pixel with specific values and in a specified spatial relationship occur in an image, and then extracting statistical measures from this matrix. The extracted features from GLCM are used to classify the texture by Back Propagation Neural Network to compare their effectiveness.
\end{abstract}

Keywords - Grey Level Co-occurrence Matrix (GLCM), Back Propagation Neural Network (BPNN)

\section{INTRODUCTION}

Textile industry plays a vital role in the present day business market. The automation process has been increasingly used in manufacturing and processing of fabrics. Despite automation there is a lot of concern for quality fabrics leading to need of efficient computer techniques. Presently, quality of fabrics is mainly done by manual process which is time consuming. The important objective in automation nowadays is to identify the defect in the woven fabrics with less manpower and in less time.

Woven fabrics are formed by interlacing of two yarns. These are warp yarn and weft yarn. Warp refers to the long vertical yarns that are wrapped around the looms. Weft refers to the horizontal yarns that are woven through the warp yarns.

The defect detection of woven fabric has been reported by many researchers [1]-[4].The proposed method consists of two steps: 1) feature extraction using GLCM and 2) the detection of defects using back propagation neural network.

\section{PROPOSED METHOD}

The proposed method flow chart for defect detection of woven fabrics are shown in Fig. 1.

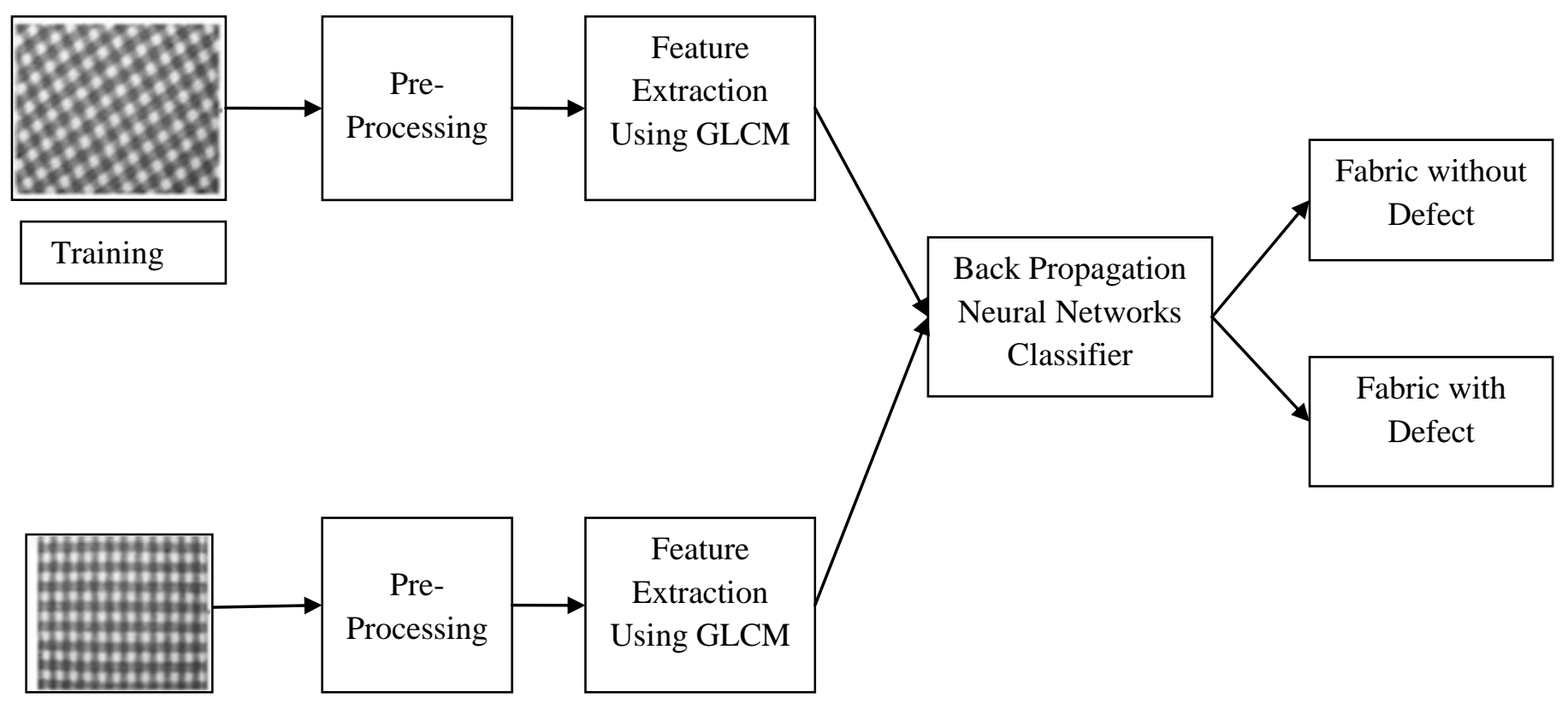

Testing

\subsection{Preprocessing}

To evaluate the proposed method, number of computersimulated and real woven material images are used. Then, the images are resized into $256 \times 256$ pixels and then converted into grayscale for increasing the processing speed.

Fig 1: Proposed method flow chart

A frequency-domain Butterworth low-pass filter is used for reducing the noise. The histogram equalization is used for further image enhancement. 


\subsection{GLCM feature extraction}

The textures may be refers to the visual impression that textures produce to human observer, which are related to local spatial variations of simple stimuli like colour, orientation and intensity in an image. In pattern recognition and in image processing, feature extraction is a special form of dimensionality reduction.

When the input data to an algorithm is too large to be processed and it is suspected to be notoriously redundant then the input data will be transformed into a reduced representation set of features also named features vector. Transforming the input data into the set of features is called feature extraction.

Basically the feature are the specific properties of pixels in the image. In this work special features are extracted using gray level co-occurrence matrix from a woven.

A co-occurrence matrix or co-occurrence distribution is a matrix or distribution that is defined over an image to be the distribution of co-occurring values at a given offset.

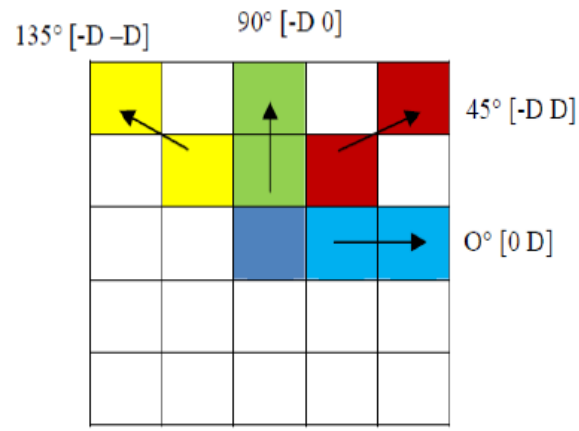

Fig 2: Example of grey level co-occurrence matrix with offset varying in distance and Orientation

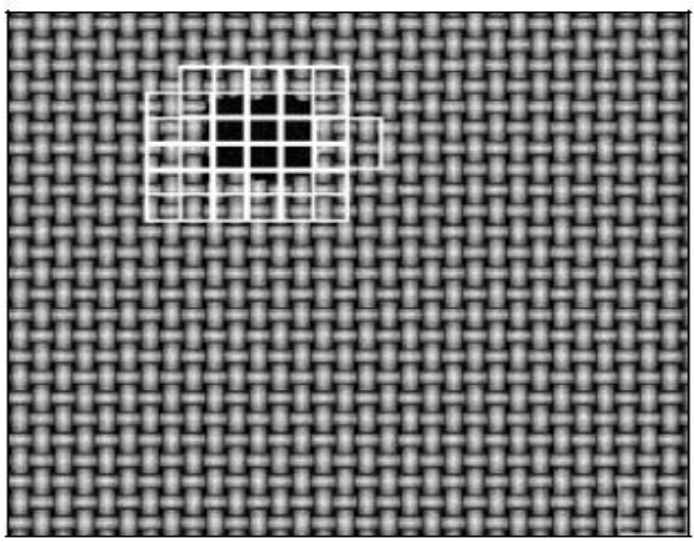

Fig 3: Example of detection of defect in woven fabric

The distance between two pixels (D) and the position angle between two pixels $(i, j)$ and $(k, l)$ i.e. $\theta$ are the parameters affect the calculation of the co-occurrence matrix. .Figure 2 shows the grey level co-occurrence matrix with offset varying in distance and Orientation. Four directions for the position angle: $\theta=0^{\circ}$ for the horizontal position, $\theta=45^{\circ}$ for the right diagonal position direction, $\theta=90^{\circ}$ for the vertical direction and $\theta=135^{\circ}$ for the left diagonal direction. All the features are calculated from the normalized co-occurrence matrices. The normalized the co-occurrence matrices can be expressed as:

$$
P_{i j}=\frac{P_{i j}}{\sum_{i=0}^{N-1} \sum_{j=0}^{N-1} P_{i j}}
$$

Haralick parameters are calculated from these co-occurrence matrices to analyze textures. For this work four parameters which are described below are used. Table 1 shows the four parameters:

Table 1: Haralick parameters

\begin{tabular}{|l|l|}
\hline Parameters & Description \\
\hline Contrast & $\begin{array}{l}\text { Measures the scale of difference in } \\
\text { the Grey level Co-occurrence } \\
\text { matrices. }\end{array}$ \\
\hline Correlation & $\begin{array}{l}\text { Measure of how correlated a pixel is } \\
\text { to its neighbor over the whole image } \\
\text { in the Grey level Co-occurrence } \\
\text { matrices. }\end{array}$ \\
\hline Entropy & $\begin{array}{l}\text { Measures the randomness of the } \\
\text { elements of the co-occurrence matrix. }\end{array}$ \\
\hline Momogeneity & $\begin{array}{l}\text { Measures the closeness of the } \\
\text { distribution of elements in the GLCM } \\
\text { to the GLCM diagonal. }\end{array}$ \\
\hline
\end{tabular}

Contrast

$$
\mathrm{CON}=\sum_{i=0}^{N-1} \sum_{j=0}^{N-1}(i-j)^{2} P_{i j}
$$

Correlation

$$
\mathrm{COR}=\sum_{i=0}^{N-1} \sum_{j=0}^{N-1} P_{i j} \frac{\left(1-\mu_{i}\right)\left(1-\mu_{j}\right)}{\sigma_{i} \sigma_{j}}
$$

Entropy

$$
\mathrm{ENT}=-\sum_{i=0}^{N-1} \sum_{j=0}^{N-1} P_{i j} \log _{2}\left(P_{i j}\right)
$$

Homogeneity

$$
\mathrm{HOM}=\sum_{i=0}^{N-1} \sum_{j=0}^{N-1} \frac{P_{i j}}{1+(i-j)^{2}}
$$

\subsection{Back Propagation Neural Network}

Neural networks are one of the fastest most flexible classifier used for fault detection due to their non-parametric nature and ability to describe complex decision regions.ANN'S are networks of interconnected computational units, usually called nodes. The input of a specific node is the weighted sum of the output of all the nodes to which it is connected. The output value of a node is, in general, a non-linear function (referred to as the activation function) of its input value. The multiplicative weighing factor between the input of node $j$ and the output of node $\mathrm{i}$ is called the weight $w_{i f}$.

Back-propagation NN's used in this study consist of one input layer, one or two hidden layers, and one output layer. With back-propagation, the input data (Extracted GLCM Features) is repeatedly presented to the Artificial Neural Network, with each presentation the output of the neural network is compared to the desired output and an error is computed. This error is then fed back (back-propagated) to the Artificial Neural Network and used to adjust the weights such that the error decreases with each iteration and the neural model gets closer and closer to producing the desired output. This process is known as Training. 
The Training of these networks consists in finding a mapping between a set of input values and a set of output values. This mapping is accomplished by adjusting the value of the weights $W_{i j}$ using a learning algorithm, the most popular of which is the generalized delta rule. After the weights are adjusted on the training set, their value isfixed and the ANN's are used to classify unknown input images. The generalized delta rule involves minimizing an error term defined as

$E_{p}=\frac{1}{2} \sum_{j}\left(t_{p j}-o_{p j}\right)^{2}$

In this work Back Propagation Neural Network includes an input layer of four input nodes, a hidden layer of twenty neurons, and an output layer of one neuron. The nonlinear transfer function is the hyperbolic tangent function with a learning speed of 0.07 , momentum coefficient of 0.7 . The operational process can be divided into the learning and the recalling stage, and there are 210 training examples and 100 testing examples. The 4 texture features extracted from the grey level co-occurrence matrix are used as the input parameters of the Back-Propagation Neural Network.

\section{RESULTS AND DISCUSSION}

Fabrics with three different texture constituting nearly 310 images are taken for analysis of proposed work. Here for texture 1, 70 images are used for training and 40 images are used for testing, for texture 2, 70 images are used for training and 30 images are used for testing and for texture 3, 70 images are used for training and 30 images are used for testing the classifier and algorithm is implemented in Matlab 7.9 .

Figure 4 shows some of the training and testing images. Four features are taken for this work as these give better results than the other parameters.

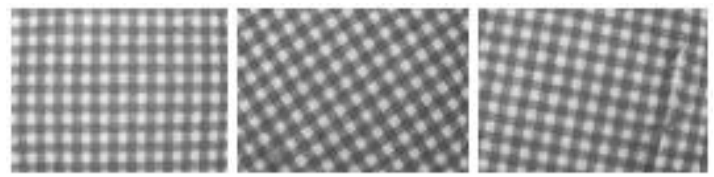

Some of texture 1 images

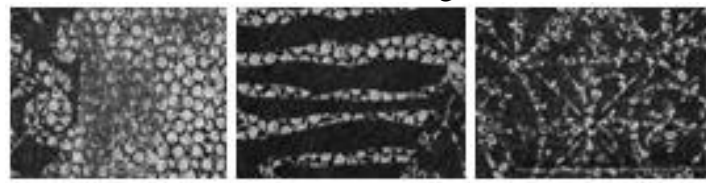

Some of texture 2 images

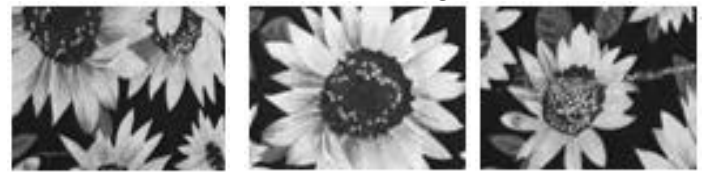

Some of texture 3 images

Fig. 4 Some of the training and testing images

Chart 1, 2 and 3 shows the comparison of four back propagation training algorithm with different combination of four GLCM features for Texture 1,2 and 3.

Accuracy: Accuracy is a statistical measure of how well a classifier correctly identifies or excludes a condition. The accuracy is the proportion of true results (both true positive and true negative) in the population.

Accuracy $=\frac{\mathrm{TP}+\mathrm{TN}}{\mathrm{TP}+\mathrm{TN}+\mathrm{FP}+\mathrm{FN}}$
The analysis is carried out using various back propagation techniques such as gradient descent (gd), gradient descent with adaptive learning rate (gda), gradient descent with momentum (gdm), gradient descent momentum and adaptive learning rate $(\mathrm{gdx})$.

Chart 1. Comparison chart for texture 1

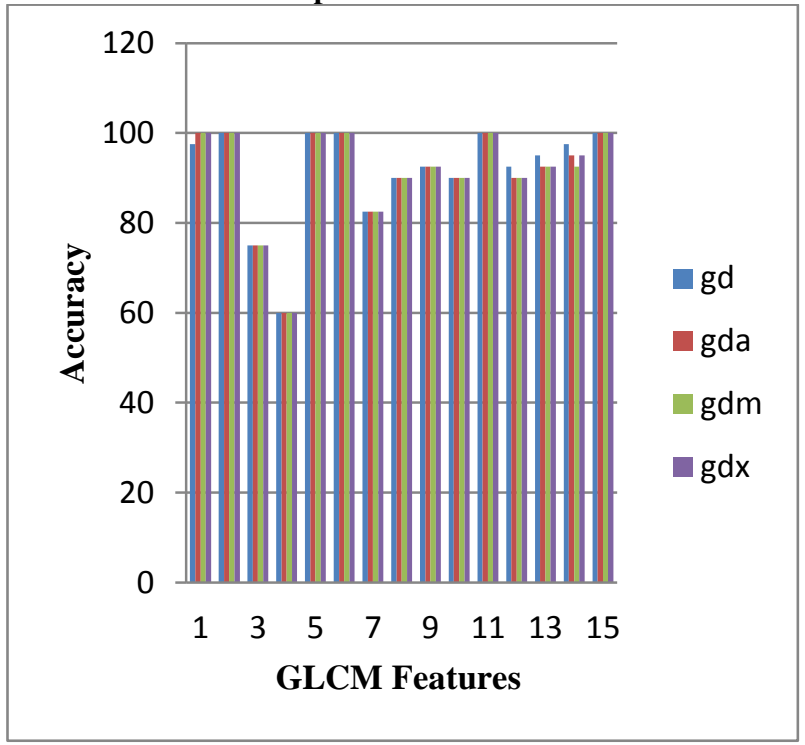

Chart 2. Comparison chart for texture 2

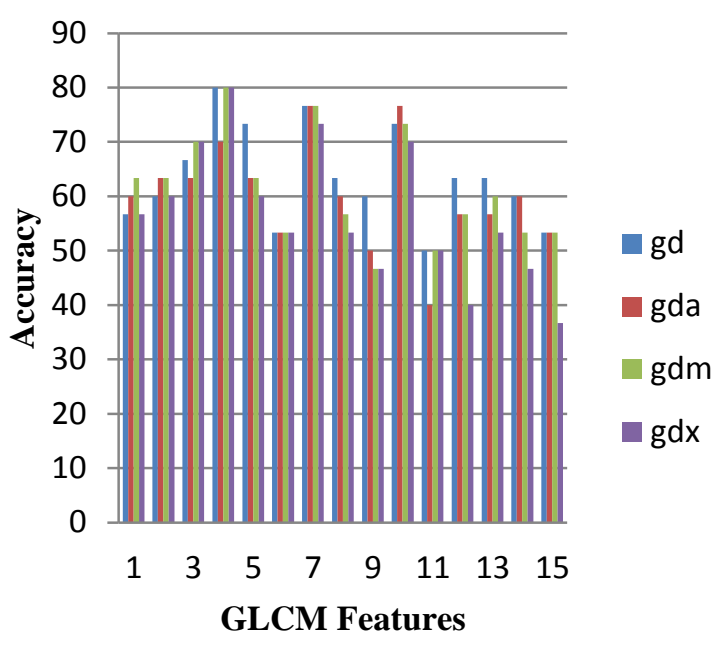


Chart 3. Comparison chart for texture 3

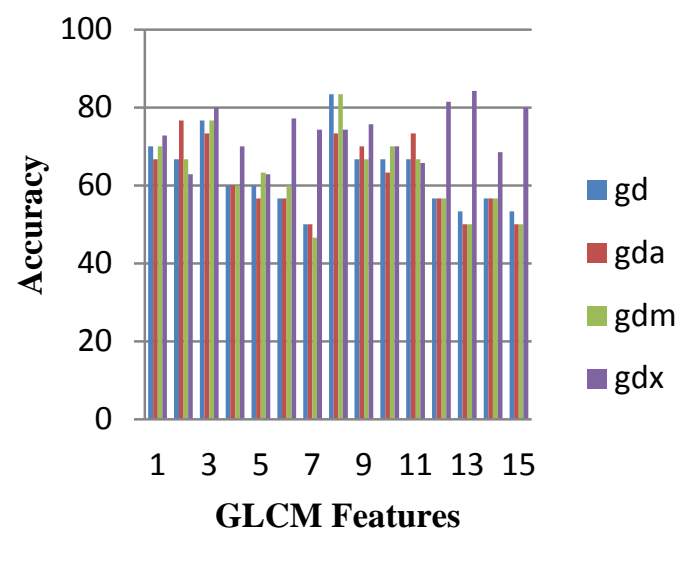

The features mentioned below are used for analysis of fabric
1. Contrast $(\mathrm{C} 1)$
2. Correlation $(\mathrm{C} 2)$
3. Entropy (E)
4. Homogeneity $(\mathrm{H})$
5. Contrast + Correlation
6. Correlation + Entropy
7. Entropy + Homogeneity
8. Contrast + Entropy
9. Correlation + Entropy
10. Contrast + Homogeneity
11. $\mathrm{C} 1+\mathrm{C} 2+\mathrm{E}$
12. $\mathrm{C} 2+\mathrm{E}+\mathrm{H}$
13. $\mathrm{C} 1+\mathrm{E}+\mathrm{H}$
14. $\mathrm{C} 1+\mathrm{C} 2+\mathrm{H}$
15. $\mathrm{C} 1+\mathrm{C} 2+\mathrm{E}+\mathrm{H}$

The Chart 1 to 3 shows the accuracy expressed in percentage for different back propagation training networks with 20 hidden layer neurons. In texture 1 , all the four training networks exhibit better performance in terms of accuracy. In texture 2, gradient descent, gradient descent with momentum and gradient descent momentum and adaptive learning rate exhibit better performance in terms of accuracy. In texture 3, gradient descent momentum and adaptive learning rate exhibits better performance in terms of accuracy.

\section{CONCLUSION}

This paper used the Back-Propagation Neural Network for the development of texture classification for recognizing fabric defects. The texture of fabrics can be characterized by the spatial co-occurrence of their color components making the GLCM a suitable method for the analysis. Four parameters like contrast, correlation, entropy, homogeneity, have been used for calculating the fabric textures. Comparing different back propagation training networks gradient descent momentum and adaptive learning rate exhibits better performance for the fabrics having different textures in terms of accuracy.

\section{REFERENCES}

[1] Dr.Mohamed Mansoor Roomi, S.Saranya, "Bayesian classification of fabrics using binary co-occurrence matrix", International Journal of Information Sciences and Techniques (IJIST) Vol.2, No.2, March 2012.

[2] Te-Li Su, Hua-Wei Chen, Gui-Bing Hong, Chih-Ming $\mathrm{Ma}$, "Automatic inspection system for defects classification of Stretch knitted fabrics", international conference on wavelet analysis and pattern recognition, qingdao, 11-14 july 2010.

[3] Yassine Ben Salem, Salem Nasri, "Texture classification of woven fabric based on a glcm Method and using multiclass support vector machine", $6^{\text {th }}$ international multi conference on systems, signals and devices, 2009.

[4] Chung-Feng Jeffrey Kuo and Ching-Jeng Lee, "A BackPropagation Neural Network for Recognizing FabricDefects" Textile Research Journal 2003; 73; 147, Feb 2003

[5] B. S. Jeon, J. H. Bae, and M. W. Suh, "Automatic recognition of woven fabric patterns by an artificial neural network," Textile Res. J., vol. 73, no. 7, pp. 645650, Jul. 2003.

[6] C. F. J. Kuo and C. C. Tsai, "Automatic recognition of fabric nature by using the approach of texture analysis," Textile Res. J., vol. 76, no. 5, pp. 375-382, May 2006.

[7] C. F. J. Kuo, C. Y. Shih, and J. Y. Lee, "Automatic recognition of fabric weave patterns by a fuzzy C-means clustering method," Textile Res. J., vol. 74, no. 2, pp. 107-111, Feb. 2004

[8] Maria Petrou, Pedro Garcia Sevilla, "Image processing dealing with Texture",Tata McGraw Hill publications, $3^{\text {rd }}$ edition.

[9] Xin Wang, Nicolas D. Georganas, Life Fellow, IEEE, and Emil M. Petriu, Fellow, IEEE, "Fabric Texture Analysis Using Computer vision techniques" IEEE transactions on instrumentation and measurement, vol. 60, no. 1, january 2011.

[10] Rafeal C.Gonzalez, "Digital image processing" Pearson Publications , $2^{\text {nd }}$ Edition, 2004 